\title{
GRBS FOLLOWED-UP BY THE BOOTES NETWORK
}

S. Guziy ${ }^{1,17}$, A. Castro-Tirado ${ }^{1}$, M. Jelínek ${ }^{1}$, J. Gorosabel ${ }^{1,21,22}$, P. Kubánek ${ }^{1}$, R. Cunniffe ${ }^{1}$, O. Lara-Gil ${ }^{1}$, O. Rabaza-Castillo ${ }^{1}$, A. de Ugarte Postigo ${ }^{1}$, R. Sánchez-Ramírez ${ }^{1}$, J. Tello ${ }^{1}$, C. Pérez del Pulgar ${ }^{3}$, S. Castillo-Carrión ${ }^{4}$, J. Castro Cerón ${ }^{5}$, T. de J. Mateo Sanguino ${ }^{6}$, R. Hudec ${ }^{7}$, S. Vitek $^{8}$, B. de la Morena Carretero ${ }^{9}$, J. Díaz Andreu ${ }^{9}$, R. Fernández-Muñoz ${ }^{10}$, D. Pérez-Ramírez ${ }^{11}$, P. Yock ${ }^{12}$, W. Allen ${ }^{13}$, I. Bond ${ }^{14}$, I. Kheyfets ${ }^{17}$, G. Christie ${ }^{15}$, L. Sabau-Graziati ${ }^{16}$, C. Cui ${ }^{18}$, Y. Fan ${ }^{19}$ and I.H. Park ${ }^{20}$

${ }^{1}$ Instituto de Astrofísica de Andalucía, IAA-CSIC, PO Box 03004, 18080 Granada, Spain

${ }^{2}$ Image Processing Laboratory, Univ. de Valencia, Burjassot, Valencia, Spain

${ }^{3}$ Departamento de Ingeniería de Sistemas y Automática, Univ. de Málaga, Spain

${ }^{4}$ Servicio Central de Informática, Universidad de Málaga, Spain

${ }^{5}$ European Space Astronomy Centre, ESAC, Villafranca del Castillo Madrid, Spain

${ }^{6}$ Departamento de Ingeniería de Sistemas y Automática, Universidad de Huelva, E.P.S. de La Rábida, Huelva, Spain

7 Astronomical Institute, Academy of Sciences of the Czech Republic, 25165 Ondřejov, Czech Republic

${ }^{8}$ Czech Technical University, Faculty of Electronic Engineering, Dep. of Radioelectronics, Czech Republic

${ }^{9}$ Estación de Sondeos Atmosféricos, ESAt de El Arenosillo, CEDEA-INTA, Mazagón, Huelva, Spain

${ }^{10}$ Estación Experimental de La Mayora, EELM-CSIC, Algarrobo Costa, Málaga, Spain

11 Universidad de Jaén, Campus Las Lagunillas, Jaén, Spain

12 University of Auckland, New Zealand

13 Vintage Lane Observatory, Blenheim, New Zealand

${ }^{14}$ Massey University, New Zealand

15 Stardome Observatory, Auckland, New Zealand

${ }^{16}$ División de Ciencias del Espacio, INTA, Torrejón de Ardoz, Madrid, Spain

17 Astronomical Observatory, Nikolaev National University, Nikolaev, Ukraine

18 National Astronomical Observatory, Chinese Academy of Sciences, Beijing 100012, China

19 Yunnan Astronomical Observatory, CAS Kunming, 650011 Yunnan, China

${ }^{20}$ Ewha Womans University, Daehyundong 11-1, Seodaemungu, 120-750 Seoul, South Korea

${ }^{21}$ Unidad Asociada Grupo Ciencia Planetarias UPV/EHU-IAA/CSIC, Departamento de Física Aplicada I, E.T.S. Ingeniería, Universidad del País Vasco UPV/EHU, Alameda de Urquijo s/n, 48013 Bilbao, Spain

${ }^{22}$ Ikerbasque, Basque Foundation for Science, Alameda de Urquijo 36-5, 48008 Bilbao, Spain 


\begin{abstract}
The Burst Observer and Optical Transient Exploring System (BOOTES), is a global robotic observatory network, which started in 1998 with Spanish leadership devoted to study optical emissions from gamma ray bursts (GRBs) that occur in the Universe. We present shot history and current status of BOOTES network. The Network philosophy, science and some details of 117 GRBs followed-up are discussed.
\end{abstract}

\title{
1 Introduction
}

The Burst Observer and Optical Transient Exploring System (BOOTES), started in 1998 as a Spanish-Czech collaboration devoted to study optical emissions from gamma ray bursts (GRBs).

The first BOOTES robotic astronomical station was located at INTA's Estación de Sondeos Atmosféricos in Centro de Experimentación de El Arenosillo, a darksky site near Mazagón (Huelva), center owned by the Instituto Nacional de Técnica Aerospacial (INTA). The second observing station was opened in 2001 and it is located at the Estación Experimental de La Mayora (dubbed BOOTES-2), $240 \mathrm{~km}$ apart. The latter is run by the Consejo Superior de Investigaciones Científicas (CSIC). In 2009 BOOTES expanded abroad, with the third station (BOOTES-3) being installed in Blenheim (South Island, New Zealand). The fourth one (BOOTES-4) has been deployed in 2011 at the Lijiang Astronomical Observatory (unnan, China).

\section{Location}

Four BOOTES station are located in the three countries (Spain, New Zealand and China) that have different geographic coordinates. In our dreams and plans an installation of three new station in the other geographic locations (countries). A general view of BOOTES stations in the world is shown in Figure 1.

\section{Science and goals}

The observation of the GRB error box simultaneously to the GRB occurrence Although the first detected optical counterparts were not brighter than $19^{\text {th }}$ mag few hours after the burst, there have been several GRBs for which optical transient emission has been detected simultaneously to the gamma-ray event, with magnitudes in the range $5-10$. The faint transient emission that has been detected few hours after the event is a consequence of the expanding remnant that the GRB leaves behind it. This provides information about the surrounding medium, but not about the burster itself. The fast slewing $0.6 \mathrm{~m}$ BOOTES telescopes should produce important results in this field.

The detection of optical flashes (OTs) of cosmic origin that could be unrelated to GRBs and constitute a new type of different astrophysical phenomenon 


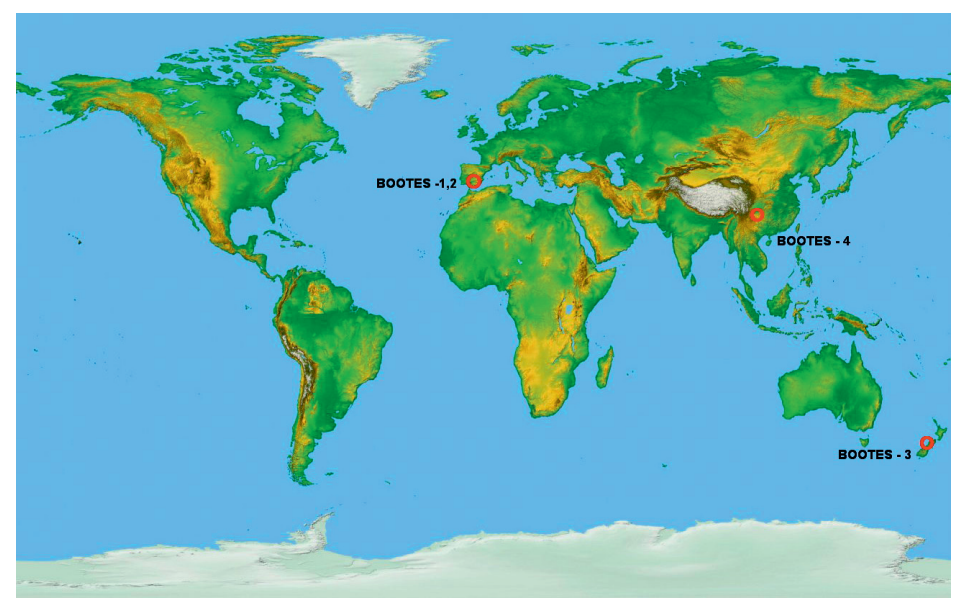

Fig. 1. The telescopes locations in the world.

(perhaps associated to QSOs/AGNs). If some of them are related to GRBs, the most recent GRB models predict that there should be a large number of bursting sources in which only transient X-ray/optical emission should be observed, but no gamma-ray emission. The latter would be confined in a jet-like structure and pointing towards us only in a few cases.

The monitoring of high-energy targets in different optical, as groundbased support for the ESA's International Gamma-Ray Laboratory (INTEGRAL) in which Spain had, for the first time, the leadership in one of the instruments, the Optical Transient Camera (OMC). This included test of technologies, methods, data processing, ground-based observational network, etc.. INTEGRAL was launched in 2002.

The monitoring of several objects (bright AGNs/QSOs, old GRB positions, etc.) looking for recurrent optical transient optical emission arising from these sources. There are hints that sudden and rapid flares occurs, though of smaller amplitude. This will be achieved by means of the $0.6-\mathrm{m}$ network of BOOTES telescopes.

\section{GRBs with BOOTES network}

GRBs are indeed one of the main scientific goals of BOOTES. We know that GRBs arise at cosmological distances (with mean redshift $z \sim 2.5$ and redshifts in the range $\sim 0.01$ to $\sim 10$ ), with huge isotropic equivalent radiated energy, and small timescales (in the range few ms to $10^{2} \mathrm{~s}$ ), thus implying a small emitting region. The spectrum is non-thermal and relativistic outflows $(\Gamma>100)$ are involved. A frequent assumption is that short and long GRBs (with the short ones representing $1 / 3$ of the overall GRB population) are due to different progenitors leading to the same succession of events: formation of a compact object and ejection of 
a relativistic outflow which produces the (long-lasting) afterglow at other wavelengths. Main program for BOOTES network system is observations optical counterparts for gamma ray burst: open and monitoring OT in different filters. The BOOTES network philosophy is: identical telescopes spaced around the Earth, identical filter sets: g'r'i'ZY, identical CCD cameras, impact on several scientific fields and public outreach, Castro-Tirado et al. (2012).

Some summary of GRB response at the BOOTES stations and examples of observations we are present below:

- BOOTES-1/0.3 m. Around 50 real-time follow-ups in 2004-2012, with 10 detections of the $\mathrm{OA}$ and 27 observations with upper limit, 12 pulications (GCN circulars and other), Jelínek et al. (2010);

- BOOTES-2/0.3 m. 24 real-time follow-ups, with 9 upper limit of the OA and 5 publications;

- BOOTES-2/0.6 m - TELMA. 8 real-time follow-ups with 3 detections of the OA, 5 observations with upper limit, 7 publications;

- BOOTES-3/0.6 m - YA. 23 real-time follow-ups with 6 detections of the OA, 10 observations with upper limit, 18 publications;

- BOOTES-4/0.6 m - MET. 12 real-time follow-ups with 1 detection of the OA, 5 observations with upper limit, 2 publications (GCN circulars).

\section{Discussion}

We have shown the advances in establishing the worldwide network of BOOTES telescopes in different locations around the Earth. BOOTES has played a significant role in the gamma-ray burst field over the last decade. Multiwavelength observations (photometry, spectroscopy, polarimetry) are ideal to better understand the GRB diversity. As of Sep. 2012, the number of GRBs followed-up at the four BOOTES stations is 117, with 20 optical counterpart detections and 56 upper limits reported (the rest being too crowd fields or unusable due to dew, low airmass, unfocused images, ...), altogether leading to 44 publications.

Installing the remaining BOOTES stations will help in continuous monitoring for some celestial sources, building more precise light curve for the targets. BOOTES contributes significantly to the GLORIA Network (EU-FP7) too. More detailed information about the BOOTES network can be seen in http://bootes. iaa.es

We acknoweledge the support of the Spanish Ministry Projects AYA 2009-14000-C03-01 and AYA 2012-39727-C03-01.

\section{References}

Castro-Tirado, A.J., Jelínek, M., Gorosabel, J., et al., 2012, BASI, in press Jelínek, M., Castro-Tirado, A.J., de Ugarte Postigo, A., et al., 2010, AdAst, id. 432172 